\title{
Three-sublattice skyrmion crystal in the antiferromagnetic triangular lattice
}

\author{
H. D. Rosales, ${ }^{1}$ D. C. Cabra, ${ }^{1,2}$ and Pierre Pujol ${ }^{3}$ \\ ${ }^{1}$ Instituto de Física de La Plata and Departamento de Física, Universidad Nacional de La Plata, CC 67, 1900 La Plata, Argentina \\ ${ }^{2}$ Abdus Salam International Centre for Theoretical Physics, Associate Scheme, Strada Costiera 11, 34151 Trieste, Italy \\ ${ }^{3}$ Laboratoire de Physique Théorique, IRSAMC, CNRS and Université de Toulouse, UPS, F-31062 Toulouse, France
}

(Received 6 August 2015; revised manuscript received 20 October 2015; published 30 December 2015)

\begin{abstract}
The frustrated classical antiferromagnetic Heisenberg model with Dzyaloshinskii-Moriya (DM) interactions on the triangular lattice is studied under a magnetic field by means of semiclassical calculations and large-scale Monte Carlo simulations. We show that even a small DM interaction induces the formation of an antiferromagnetic skyrmion crystal (AF-SkX) state. Unlike what is observed in ferromagnetic materials, we show that the AF-SkX state consists of three interpenetrating skyrmion crystals (one by sublattice), and most importantly, the AF-SkX state seems to survive in the limit of zero temperature. To characterize the phase diagram we compute the average of the topological order parameter which can be associated with the number of topological charges or skyrmions. As the magnetic field increases this parameter presents a clear jump, indicating a discontinuous transition from a spiral phase into the AF-SkX phase, where multiple Bragg peaks coexist in the spin structure factor. For higher fields, a second (probably continuous) transition occurs into a featureless paramagnetic phase.
\end{abstract}

\section{INTRODUCTION}

Twisted modulated magnetic textures due to an antisymmetric spin exchange interaction, termed the DzyaloshinskiiMoriya interaction, have attracted much interest mainly after the experimental observation of nontrivial magnetic configurations, called magnetic skyrmion lattices, which have important potential technological applications [1]. Recently, strong evidence of the formation of a skyrmion crystal (SkX) state was observed in the metallic ferromagnet $\mathrm{MnSi}$ [2]. This state consists of a triangular lattice arrangement of skyrmions and can be visualized as a superposition of three nonequivalent spiral states (each characterized by one wave vector $\mathbf{k}$ ) [3-8]. In systems with a square lattice structure, the SkX phase arises from the competition of the ferromagnetic and the Dzyaloshinskii-Moriya (DM) interactions and it is stabilized by a magnetic field and thermal fluctuations. Recently, Okubo et al. have shown that it is also possible to stabilize the SkX phase in the isotropic Heisenberg model in the triangular lattice with strong nearest-neighbor (ferromagnetic) and weak next-nearest-neighbor (antiferromagnetic) interactions [9].

In this paper, based on analytical approximations and Monte Carlo simulations, we show that in a pure antiferromagnetic frustrated system it is possible to stabilize a phase closely related to the SkX phase described above. The state found in the present paper consists of three interpenetrating skyrmion lattices (one by sublattice) that we call antiferromagnetic SkX (AF-SkX). This phase survives even in the limit $T \rightarrow 0$ and can be stabilized for small values of $D / J$. To identify and characterize this phase we compute a chiral order parameter which determines the density of skyrmions present in the lattice. Due to the discrete nature of the model, in the region of large magnetic fields a series of discrete jumps in the order parameter is observed.

The paper is organized as follows: In Sec. II we present the Hamiltonian and study the classical ground state solutions at zero magnetic field and zero temperature based on the spherical approximation. This analysis shows that the ground state has a threefold degeneracy which could be a source of exotic ordered states that might be realized under applied fields, e.g., various types of states where multiple wave vectors coexist (so-called multiple- $q$ states). Section III contains our Monte Carlo results. We found a quite rich low-temperature behavior of the system as the magnetic field is varied. The system goes from a spiral low-field phase to an antiferromagnetic skyrmion lattice phase at higher field. Finally, increasing the magnetic field further, the system enters into a featureless paramagnetic phase. We pay particular attention to the undoubtedly most interesting phase which is the antiferromagnetic skyrmion lattice phase. It has the particularity of being composed of three interpenetrating and shifted skyrmion lattices that are realized on each sublattice of the triangular lattice. The Monte Carlo (MC) study is complemented in Sec. IV by a phenomenological analysis and in Sec. V we analyze the stability of the skyrmion phase and briefly discuss the relation of the present study with the square lattice analog. We conclude in Sec. VI with a summary and discussion of our results.

\section{MODEL AND SPHERICAL APPROXIMATION}

Let us consider the antiferromagnetic Heisenberg model on the triangular lattice in a magnetic field, with the Hamiltonian given by

$$
\mathcal{H}=J \sum_{\left\langle\mathbf{r}, \mathbf{r}^{\prime}\right\rangle} \mathbf{S}_{\mathbf{r}} \cdot \mathbf{S}_{\mathbf{r}^{\prime}}+D \delta \hat{\mathbf{r}} \cdot\left(\mathbf{S}_{\mathbf{r}} \times \mathbf{S}_{\mathbf{r}^{\prime}}\right)-h \sum_{\mathbf{r}} S_{\mathbf{r}}^{z},
$$

where the spin variables are unimodular classical vectors, $\left|\mathbf{S}_{\mathbf{r}}\right|=1, J>0$ and $D$ are respectively the antiferromagnetic and DM couplings, $\delta \hat{\mathbf{r}}=\left(\mathbf{r}^{\prime}-\mathbf{r}\right) /\left|\mathbf{r}^{\prime}-\mathbf{r}\right|$ is a unitary vector pointing along the axis, $\sum_{\left\langle\mathbf{r}, \mathbf{r}^{\prime}\right\rangle}$ means the sum over nearest-neighbor $(\mathrm{NN})$ couplings on the triangular lattice with primitive translation vectors of the direct lattice $\vec{e}_{1}=(1,0)$ and $\vec{e}_{2}=(1 / 2, \sqrt{3} / 2)$, and $h$ the strength of the magnetic field along the $z$ axis. The DM interaction is chosen in the direction of the $\delta \hat{\mathbf{r}}$ vectors, so as to give rise to spiral spin states with the spins lying in a plane perpendicular to the propagation vector [5]. 


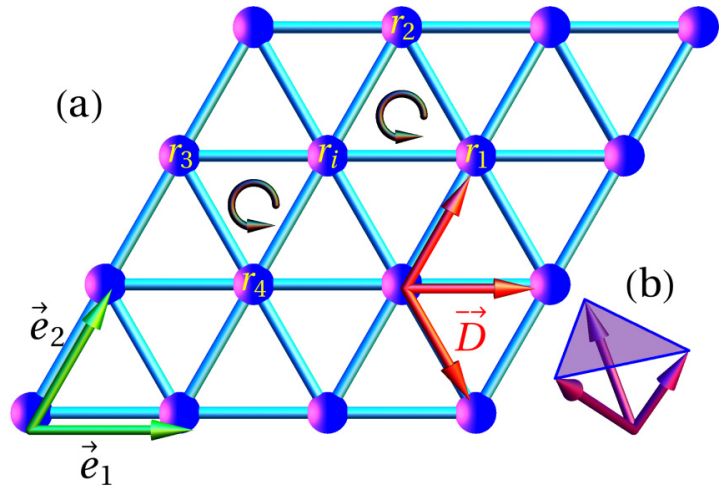

FIG. 1. (Color online) (a) Triangular lattice. The green arrows indicate the primitive translation vectors of the direct lattice $\vec{e}_{1}=(1,0)$, $\vec{e}_{2}=(1 / 2, \sqrt{3} / 2)$, red arrows the Dzyaloshinskii-Moriya vectors. The sites with labels $\mathbf{r}_{i}, \mathbf{r}_{1}, \ldots, \mathbf{r}_{4}$ indicate the sites involved in the calculation of the local chirality. (b) Area of the triangle to compute for the discretized skyrmion number $A_{\mathbf{r}}^{a b}$ [Eq. (8)].

The magnetic phase diagram for the model defined by Eq. (1) with $D=0$ has been discussed in Refs. $[10,11]$. At $T=0$ and for zero magnetic field, the system orders in a $120^{\circ}$ three-sublattice magnetic structure described by the wave vector $\mathbf{k}=(4 \pi / 3,0)$. In a magnetic field the classical energy is minimized for spin configurations constrained by the magnetization of each triangular plaquette:

$$
\mathbf{S}_{\triangle}=\mathbf{h} /(3 J) .
$$

This constraint leaves undetermined the orientation of the spin plane and sublattice directions inside that plane. This degeneracy persists up to the saturation field $h_{s}=9 \mathrm{~J}$. However, due the order-from-disorder effect [12] this degeneracy is lifted by thermal fluctuations, which select kinds of states (collinear and coplanar) over the non-coplanar ones at low temperature. The resulting phases depend on the magnetic field strength: for low field a coplanar so-called Y state, with one spin pinned in the negative $z$ direction and two canted up; at exactly $h / J=3$ the pseudo-plateau-state (at $M=1 / 3$ ) with the collinear configuration up-up-down; and in the high-field region a coplanar canted version of it, which smoothly interpolates with the fully polarized state at $h / J=9$.

Now we turn-on the DM interaction with the orientation along the nearest-neighbor bonds (Fig. 1). As a first step we investigate the magnetic order of the Hamiltonian Eq. (1) at zero magnetic field and zero temperature by means of the spherical approximation [13]. Within this scheme, instead of imposing the local length constraint $\left|\mathbf{S}_{\mathbf{r}}\right|=1$, one imposes a milder condition, $\sum_{\mathbf{r}}\left|\mathbf{S}_{\mathbf{r}}\right|^{2}=N S^{2}$, where $N$ is the number of lattice sites. With this softer constraint, the model Hamiltonian (1) can be diagonalized by a simple Fourier transformation $S_{\mathbf{r}, a}^{\alpha}=\sum_{\mathbf{k}} S_{\mathbf{k}, a}^{\alpha} e^{i \mathbf{r} \cdot \mathbf{k}}$. Here, the index $\alpha=x, y, z$ is the spin component, $a=1,2,3$ is the sublattice label, and $\mathbf{r}$ and $\mathbf{k}$ denote the position and pseudomomentum, respectively. The Hamiltonian becomes

$$
\begin{aligned}
\mathcal{H} & =\sum_{\mathbf{k}} \Psi_{-\mathbf{k}} \cdot \mathbf{M}(\mathbf{k}) \cdot \Psi_{\mathbf{k}}, \\
\Psi_{\mathbf{k}} & =\left\{S_{\mathbf{k}, 1}^{x}, S_{\mathbf{k}, 1}^{y}, S_{\mathbf{k}, 1}^{z}, S_{\mathbf{k}, 2}^{x}, S_{\mathbf{k}, 2}^{y}, S_{\mathbf{k}, 2}^{z}, S_{\mathbf{k}, 3}^{x}, S_{\mathbf{k}, 3}^{y}, S_{\mathbf{k}, 3}^{z}\right\},
\end{aligned}
$$

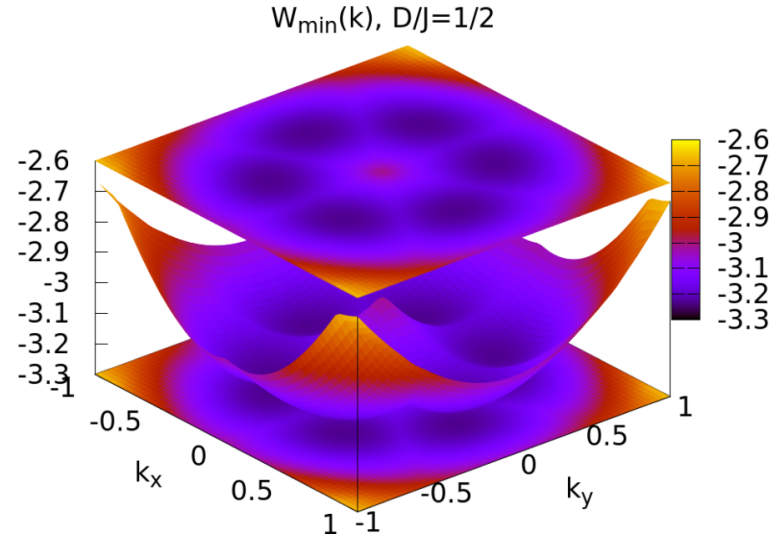

FIG. 2. (Color online) Minimum eigenvalue $w^{\min }(\mathbf{k})$ in the spherical approximation for $D / J=1 / 2$. For any value of $D / J>0$ we find that the ground state corresponds to a triple- $q$ state by sublattice.

where the $9 \times 9$ matrix

$$
\mathbf{M}(\mathbf{k})=\left[\begin{array}{ccc}
0 & m_{12} & m_{13} \\
m_{12}^{*} & 0 & m_{32}^{*} \\
m_{13}^{*} & m_{32} & 0
\end{array}\right]
$$

and the $3 \times 3$ matrices $m_{a b}$ depend on $J, D$, and $\mathbf{k}$ with explicit expressions given in the Appendix.

Since we are at $T=0$, the ground state is obtained from the minimum eigenvalue $w^{\min }(\mathbf{k})$ of the matrix $\mathrm{M}(\mathbf{k})$ [Eq. (4)]. In particular, we find that for any value of $D / J>0$ there are three minima (see Fig. 2) suggesting the presence of multiple$q$ states. In this configuration the ordering wave vectors $\mathbf{k}^{*}$ appear along the directions of the nearest-neighbor bonds with module

$$
\left|\mathbf{k}^{*}\right| \simeq \frac{D}{J}+\frac{\sqrt{3} D^{2}}{4 J^{2}}-\frac{D^{3}}{4 J^{3}}-\frac{7 \sqrt{3} D^{4}}{32 J^{4}}+O\left(\left[\frac{D}{J}\right]^{5}\right) .
$$

In particular we are interested in the nontrivial triple- $q$ state on each sublattice, which consists of a superposition of three spirals (each characterized by one wave vector $\mathbf{k}^{*}$ ), that corresponds to the skyrmion lattice phase. Within the spherical approximation, performed at both zero temperature and zero magnetic field, we find it conceivable that, at very low temperatures, one may neglect all wave vectors other than the three critical modes $\mathbf{k}^{*}$ and the uniform $\mathbf{k}=0$ mode. Henceforth, we expect that it maybe possible to find ordered states characterized by the number of wave vectors. In order to elucidate whether multiple- $q$ states could be stabilized at finite temperature and magnetic field we explore the behavior of the system by means Monte Carlo simulations in the next section.

\section{MONTE CARLO SIMULATIONS}

Monte Carlo simulations are performed based on the standard heat bath method combined with the overrelaxation method. Periodic boundary conditions were implemented for $N=L^{2}$ site clusters with $L=12-60$ and $L=72,96$ for some calculations. The system is gradually cooled down from high temperature. A run at each magnetic field or temperature contains typically $(0.1-1) \times 10^{6}$ Monte Carlo steps (MCSs) 
(i) Spiral

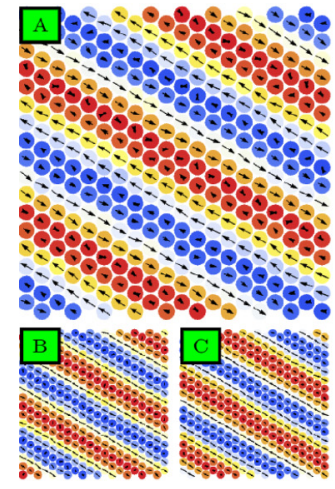

(ii) AF-SkX

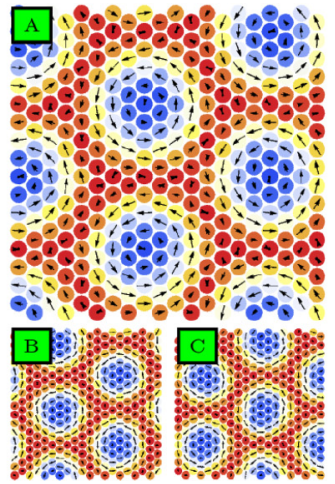

(iii) high-field

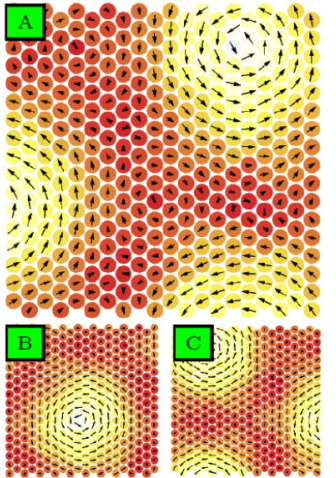

(iv) AF-SkX phase: three sublattice

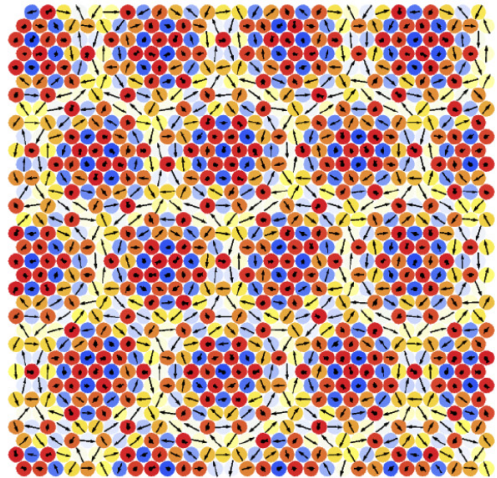

FIG. 3. (Color online) Snapshots by sublattice for $L=48, D / J=1 / 2, T / J=9 \times 10^{-3}$, and $h / J=1$ (i), 2.4 (ii), and 6.2 (iii). The green box indicates which sublattice is plotted (A, B, or C). In panel (iv) we show all three sublattices together.

for initial relaxation and twice the MCSs during the calculation of mean values.

The different phases can be easily identified from the observation of real-space spin textures (e.g., see Fig. 3). We have also computed the average of various physical quantities in order to identify precisely the different phases and the corresponding transitions between them. We first calculate the standard magnetization, magnetic susceptibility, and specific heat:

$$
\begin{gathered}
M=\frac{1}{N}\left\langle\sum_{\mathbf{r}} S_{\mathbf{r}}^{z}\right\rangle, \quad \chi_{z}=\left\langle\frac{d M}{d h}\right\rangle, \\
C=\frac{\left\langle E^{2}\right\rangle-\langle E\rangle^{2}}{N T^{2}} .
\end{gathered}
$$

We also introduce the discretized skyrmion number $\chi_{Q}$ and the total chirality $[14,15] \chi_{L}$, defined as

$$
\begin{gathered}
\chi_{Q}=\frac{1}{4 \pi}\left\langle\sum_{\mathbf{r}_{i}} A_{\mathbf{r}_{i}}^{(12)} \operatorname{sgn}\left[\chi_{L, \mathbf{r}_{i}}^{(12)}\right]+A_{\mathbf{r}_{i}}^{(34)} \operatorname{sgn}\left[\chi_{L, \mathbf{r}_{i}}^{(34)}\right]\right\rangle, \\
\chi_{L}=\frac{1}{8 \pi}\left\langle\sum_{\mathbf{r}_{i}} \chi_{L, \mathbf{r}_{i}}^{(12)}+\chi_{L, \mathbf{r}_{i}}^{(34)}\right\rangle
\end{gathered}
$$

where $A_{\mathbf{r}_{i}}^{(a b)}=\left\|\left(\mathbf{S}_{\mathbf{r}_{a}}-\mathbf{S}_{\mathbf{r}_{i}}\right) \times\left(\mathbf{S}_{\mathbf{r}_{b}}-\mathbf{S}_{\mathbf{r}_{i}}\right)\right\| / 2$ is the local area of the surface spanned by three spins on every elementary triangle $\mathbf{r}_{i}, \mathbf{r}_{a}, \mathbf{r}_{b}$ [see Fig. 1(b)]. Here $\chi_{L, \mathbf{r}_{i}}^{(a b)}=\mathbf{S}_{\mathbf{r}_{i}} \cdot\left(\mathbf{S}_{\mathbf{r}_{a}} \times \mathbf{S}_{\mathbf{r}_{b}}\right)$ is the so-called local chirality and $\mathbf{r}_{i}, \mathbf{r}_{1} \sim \mathbf{r}_{4}$ are the sites involved in the calculation of $\chi_{Q}$ (see Fig. 1). It is important to note that for slowly varying spin configurations, $\chi_{Q}$ and $\chi_{L}$ would coincide. In the case at hand the use of $\chi_{Q}$ turns out to be more effective because in the discrete case it approximates much better the directed area of the sphere surface, therefore providing a much better measure of the skyrmion number. We have computed the discretized skyrmion number by sublattice and so the sites $\mathbf{r}_{i}, \mathbf{r}_{a}, \mathbf{r}_{b}$ run in one sublattice. However, the value of the local chirality without sublattice distinction is also an important quantity within the context of the anomalous Hall effect. Indeed, electrons moving in the antiferromagnetic background of our system, and within the framework of the adiabatic approximation, would feel an effective flux per plaquette which is given by $\chi_{L}[14,15]$, computed between sites of the three different sublattices.

With the help of $\chi_{Q}$ we can detect the skyrmion phase as a function of the magnetic field as shown in Fig. 4 (center). At low magnetic field $h<h^{*}(T), \chi_{Q}=0$ due to the spiral configuration. Then for $h^{*}(T)<h<h^{* *}(T)$ a nonzero value of $\chi_{Q}$ indicates the presence of a wide AF-SkX phase. For instance, in this region and for a system size of $N=48^{2}$ the discretized skyrmion number in each sublattice $\chi_{Q} / N_{c} \sim 12 / N_{c}=1.5 \times 10^{-2}\left(N_{c}=N / 3\right)$, which is in perfect agreement with the number of skyrmions that can be directly observed from the MC snapshots (in Fig. 3 only a portion of the total lattice is shown). We also plot $\chi_{L}$ together with $\chi_{Q}$ showing that $\chi_{Q}$ is a better measure of the skyrmion number. We observe that the discretized skyrmion number is almost constant in the complete region and decays in a few steps as a consequence of the discrete nature of the skyrmion patterns. In this region of the phase diagram all the spins have a $z$ component greater than zero and its $x y$ components look like an array of $2 \mathrm{D}$ vortices, a pattern that can be interpreted as the remnant of the skyrmion lattice. The stepwise decrease of the skyrmion number should be associated with the finite size of the lattice, which, due to the periodic boundary conditions imposed, can accommodate only definite numbers of skyrmions. In the continuum limit we expect that this number will smoothly go to zero. In order to test this idea, we have performed an approximate (continuum limit) computation of the skyrmion radius as a function of the magnetic field, which leads to a smooth decrease of the skyrmion density (see Sec. IV).

In all the MC simulations the spin configurations have a three-sublattice structure, so in order to understand better the underlying structure, we plot the patterns by sublattice, $A, B$, and $C$. As an example, in Fig. 3 we show representative spin configurations for $L=48$ (only a small region of the entire lattice is shown to illustrate the spin texture) at three different magnetic fields: $h / J=$ (i) 1 , (ii) 2.4 , and (iii) 6.2. It should be stressed that given one of the skyrmion lattices in a given sublattice, the other two SkXs are simply translations of it by the triangular lattice primitive vectors $(1,0),(1 / 2, \sqrt{3} / 2)$ by an amount $\lambda / \sqrt{3}$, where $\lambda$ is the $\operatorname{SkX}$ wavelength.

We have also calculated the static spin structure factor in the reciprocal lattice to identify the Bragg peaks that characterize 


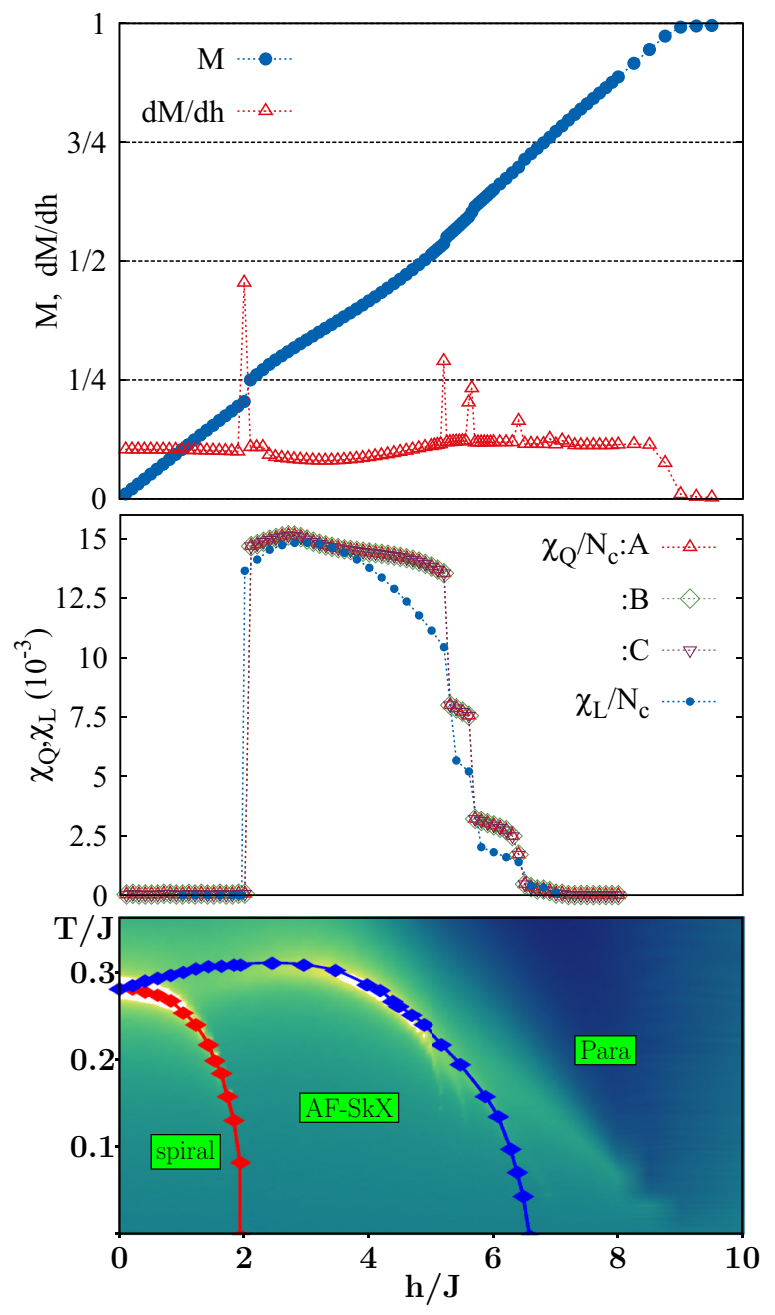

FIG. 4. (Color online) Top: Magnetization $M$ (blue) and magnetic susceptibility vs magnetic field $h$ for $L=48, D / J=0.5$, and $T / J=9 \times 10^{-3}$. Center: Discretized skyrmion number $\chi_{Q}$ and total chirality $\chi_{L}$, both quantities per sublattice, vs $h / J$ for $L=48$, $D / J=1 / 2$. Red triangles, green rhombus, and purple triangles indicate the discretized skyrmion numbers $A, B$, and $C$. Blue dots show the total chirality. Bottom: Complete $h-T$ phase diagram. The limit of the phases is obtained by the peaks in the specific heat and from the changes in the chirality order parameter.

the different spin textures. The perpendicular and parallel (to $z$ ) components $S_{\perp}(\mathbf{q})$ and $S_{\|}(\mathbf{q})$ are defined as

$$
\begin{gathered}
S_{\perp}(\mathbf{q})=\frac{1}{N}\left\langle\left|\sum_{\mathbf{r}} S_{\mathbf{r}}^{x} e^{-i \mathbf{q} \cdot \mathbf{r}}\right|^{2}+\left|\sum_{\mathbf{r}} S_{\mathbf{r}}^{y} e^{-i \mathbf{q} \cdot \mathbf{r}}\right|^{2}\right\rangle, \\
S_{\|}(\mathbf{q})=\frac{1}{N}\left\langle\left|\sum_{\mathbf{r}} S_{\mathbf{r}}^{z} e^{-i \mathbf{q} \cdot \mathbf{r}}\right|^{2}\right\rangle,
\end{gathered}
$$

where $\langle>$ means the averaged MC configurations. In Fig. 5 we show the intensity of the spin structure factor for the patterns found (Fig. 3): spiral or single- $q$ (top), AF-SkX or triple- $q$ (center), and high-field phase (bottom).

(i) Spiral phase [Fig. 3(a)]: the spin structure consists of three interpenetrating spirals on each sublattice, $A, B$, and $C$ (see Fig. 1). Each one is characterized by one of the three possible ordering wave vectors $\mathbf{k}^{*}$ obtained with the spherical
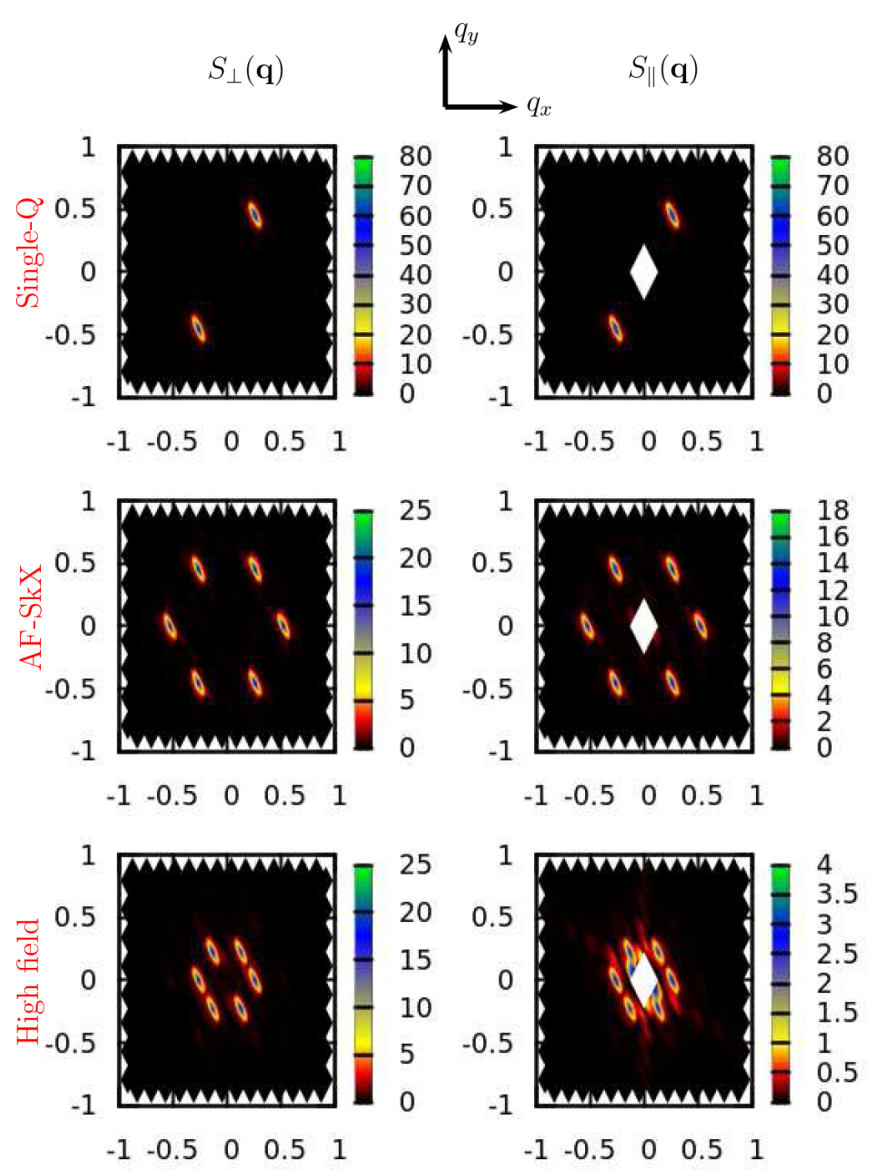

FIG. 5. (Color online) Intensity plot of the static spin structure factor for $L=96$ for the spiral phase (top), AF-SkX phase (center), and disordered phase (bottom).

approximation [Eq. (5) and Fig. 2]. Both the $x y$ and the $z$ components are characterized by the same wave vector as can be seen from Fig. 5.

(ii) Antiferromagnetic skyrmion lattice phase [Fig. 3(b)]: here the stable phase corresponds to three SkX phases, one on each sublattice. Each of the SkX phases is a superposition of three spirals characterized, both the $x y$ and the $z$ components, by the three wave vectors $\mathbf{k}^{*}$. The complete superposition of these three sublattice structures is shown in Fig. 3(d).

(iii) High-field phase [Fig. 3(c)], which in some areas shows a vortex-like structure. There, the $x y$ components of the spins form vortices while the $z$ component is always positive. This phase is established near the right edge (higher field sector), where the chirality order parameters, both $\chi_{Q}$ and $\chi_{L}$, show smaller plateaus.

The main result of our study is summarized in the phase diagram shown in Fig. 4 (bottom), which was obtained for $D / J=1 / 2$ (other values of this ratio lead to a similar phase diagram). The AF-SkX phase is surrounded by the spiral and paramagnetic phase and the lines separating the phases were obtained in different ways, locating the peaks in the specific heat (Fig. 6), the peaks in the magnetic susceptibility [Fig. 4 (top)], and using $\chi_{Q}$ as the order parameter [Fig. 4 (center)].

A very interesting behavior is observed when the system goes from the AF-SkX phase to a region where the $X Y$ components of the spins form a vortex-like structure. In all the AF-SkX region, as we increase the magnetic field, 


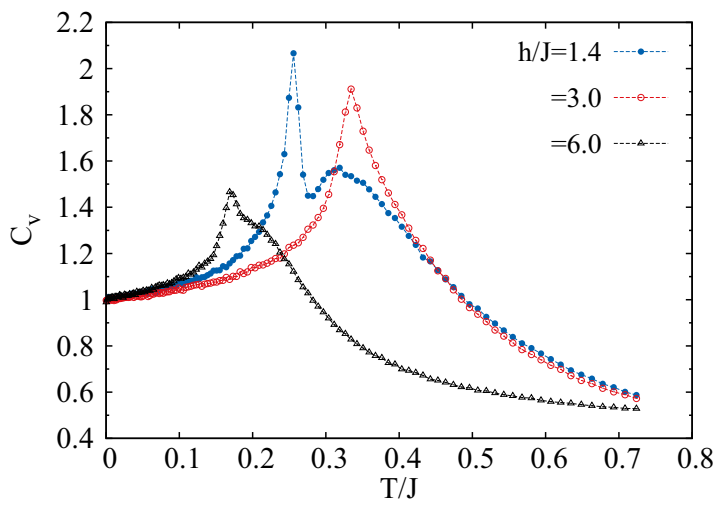

FIG. 6. (Color online) Specific heat vs temperature for $h / J=$ $1.4,3$, and 6 . The positions of the peaks are used to locate the lines separating the different phases.

the skyrmion number does not change. To increase the magnetization, the Zeeman term tends to align the spins; then in a big region of the phase diagram, antiferromagnetic exchange and DM interactions favor the AF-SkX phase with a specific topological number (the number of skyrmions). If we continue increasing the magnetic field, the system (being discrete and in a phase with a given number of skyrmions) cannot increase the magnetization without paying a high energetic cost; thus what is observed is that the AF-SkX phase changes the number of skyrmions. This result is shown in Fig. 4 (center), where both the discretized skyrmion density and total chirality density decrease by steps.

In the next section we use a phenomenological-variational analysis to compute the dependence of the skyrmion radius with the magnetic field.

\section{PHENOMENOLOGICAL ANALYSIS OF THE SKYRMION LATTICE SPACING}

Although in the MC data the number of skyrmions for a given system size, and hence the lattice spacing, varies with the applied magnetic field by discrete jumps, we suspect this behavior to be a finite-size effect. In the thermodynamic limit one can expect a lattice spacing continuously varying as a product of the competition between the applied magnetic field which tends to increase the magnetization by making bigger skyrmions and the DM interaction for which a fixed skyrmion size is optimal. To model this competition, we follow the same idea developed by Han et al. [16] in the case of a ferromagnetic system. Of course here we have a three-sublattice skyrmion pattern so the phenomenological description of a free energy in terms of spin orientations that we present has to be interpreted as representative of an average free energy for one of the three sublattices.

Consider the local spin orientation $(\theta, \phi)$ of a single skyrmion depending on the local coordinate $(r, \phi)$ as $\phi=$ $\varphi-\pi / 2$ and $\theta=\theta(r)$. The total free energy of a single skyrmion then reads

$$
\begin{aligned}
F_{S k}= & 4 \pi J \int r d r\left[\left(\frac{1}{2} \frac{d \theta}{d r}+\kappa\right)^{2}-\kappa^{2}+\frac{\kappa}{2 r} \sin (2 \theta)\right. \\
& \left.+\frac{\sin ^{2}(\theta)}{4 r^{2}}-\eta[\cos (\theta)-1]\right],
\end{aligned}
$$

where $\eta=h /(2 J)$ and $\kappa=D /(2 J)$. The function $\theta(\mathbf{r})$ minimizing the functional (12) describes the magnetization distribution in an isolated skyrmion. To understand the observed transitions phenomenologically, we calculate the energy of a isolated skyrmion using the following trial function:

$$
\begin{aligned}
\theta(r) & =\pi, \quad r \leqslant R_{0}, \\
& =\pi \frac{1-\frac{r-R_{0}}{R-R_{0}}}{1+b \frac{r-R_{0}}{R-R_{0}}}, \quad R_{0}<r \leqslant R, \\
& =0, \quad R<r .
\end{aligned}
$$

In the definition of $\theta(r)$, the parameter $R_{0}>0$ is the minimum radius simulating the central spin in the continuous limit, $R>R_{0}$ is the radius of the skyrmions, and $b$ is a parameter to be computed self-consistently. The parameters $R, b$ are fixed by the condition of minimum, $\left\{d F_{S k X} / d R, d F_{S k X} / d b\right\}=$ $\{0,0\}$. The skyrmion lattice observed in our simulations (in each sublattice) corresponds to a close packing of individual skyrmions in a triangular lattice. We then write the total free energy of the skyrmion lattice state as

$$
F_{S k X}=3 \frac{L^{2}}{2 \sqrt{3} R^{2}} F_{S k},
$$

where $L$ is the size of the system. In Fig. 7 we show a few plots of $F_{S k X}$ as a function of parameter $R$, i.e., as a function of the skyrmion radius. We observe that the behavior of the optimal skyrmion spacing as a function of the magnetic field varies very slowly for small values of the magnetic field and then behaves much more abruptly when approaching the highfield paramagnetic phase. In a fixed finite size sample, where the skyrmion lattice has to be commensurate with the sample size, this translates precisely into a large zone of the phase

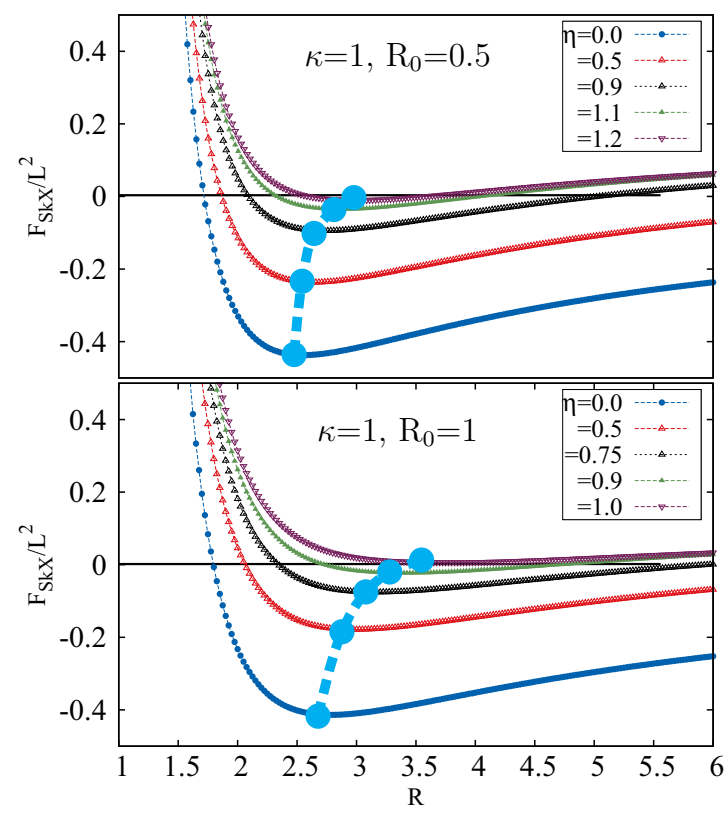

FIG. 7. (Color online) Dependence of the free energy density given by Eq. (16) as a function of the skyrmion radius $R$ for two values of the skyrmion core: $R_{0}=0.5,1(\kappa=1)$. 
diagram in which the skyrmion number is fixed and then starts to produce jumps that appear more and more abruptly.

\section{SKYRMION PERSISTENCE AND THE IMPORTANCE OF THE LATTICE}

It is well known that skyrmions are topological defects with an infinite lifetime in the continuum and with a very long persistence time in ferromagnetic lattice models. Here we are going to argue that despite having an apparently more fragile three-sublattice structure, the skyrmion persistence is also quite noticeable. To this end we compare the SkX with the total magnetization $M$ lifetimes. We start from a configuration, like Fig. 3 in the AF-SkX phase, obtained by annealing at fixed magnetic field. In Fig. 8, we show an example of the MC time evolution (the curves are the average of 500 copies) of such initial state in our simulations after a quench to zero magnetic field. Note that unlike what is observed with the normalized magnetization $M(\times 0.05$ to use the same scale for all quantities), the total chirality $\chi_{L}$ and discretized skyrmion number by sublattices $\chi_{Q}$ exhibit greater stiffness extending over several decades of time.

To complete the analysis we have also performed MC simulations in the nonfrustrated square lattice where in contrast to the case of the triangular lattice, $\delta \hat{\mathbf{r}}=\left(\mathbf{r}^{\prime}-\mathbf{r}\right) /\left|\mathbf{r}^{\prime}-\mathbf{r}\right|$ is a unitary vector pointing along the axis $\vec{e}_{1}=(1,0)$ and
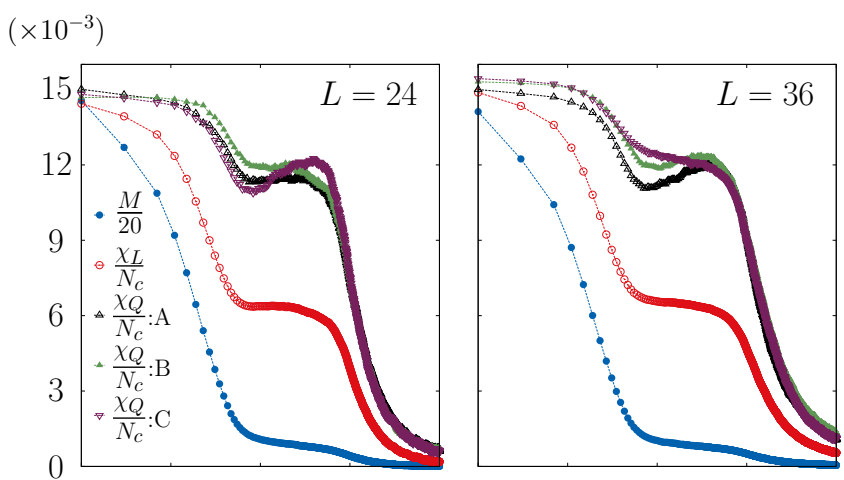

$\left(\times 10^{-3}\right)$
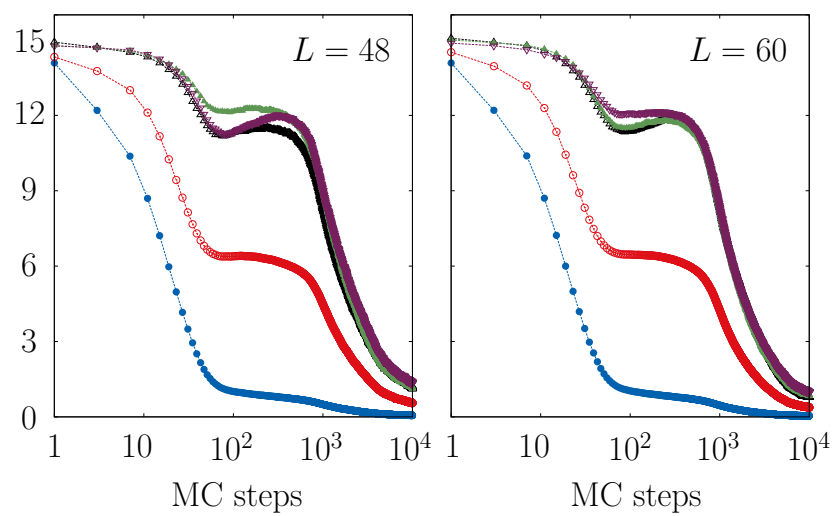

FIG. 8. (Color online) The Monte Carlo time evolution of the magnetization, discretized skyrmion number $\chi_{Q}$, and total chirality $\chi_{L}$ defined by Eqs. (8) and (9), calculated at $T=9 \times 10^{-3} J$ for sizes of $L=24,36,48,60$. The initial state corresponds to the AFSkX phase (Fig. 3). Each curve corresponds to an average of 500 independent time evolutions. $\vec{e}_{2}=(0,1)$. Our simulations do not show any evidence of an antiferromagnetic skyrmion lattice in the square lattice case. One possible explanation for the absence of this phase is geometrical frustration. At the microscopic level, on the square lattice, spins have the tendency to form a two sublattice structure, instead of three for the triangular lattice. On the other hand, at the skyrmion lattice scale, the energetically favored lattice is triangular, which fits perfectly well with a three-sublattice structure but produces frustration in the two-sublattice case (if one sublattice arranges in a triangular array, the other sublattice has to fit in a hexagonal array, which is energetically less favorable). As such, and in contrast to the ferromagnetic interactions case, the choice of the lattice seems crucial for the formation of the SkX phase.

\section{CONCLUSIONS}

In this paper we have studied the triangular lattice antiferromagnet with classical spins in the presence of inversion symmetry breaking interactions and in the presence of an external magnetic field, in order to investigate the possible appearance of skyrmion lattice structures in a pure antiferromagnetic system. We have run extensive Monte Carlo simulations complemented by simple analytical techniques. The most interesting outcome is the stabilization of a state which we term "antiferromagnetic skyrmion lattice" (AF-SkX), which consists of three interpenetrating SkX states of the usual type observed in many ferromagnetic models. This phase is stable for a wide range of magnetic fields and couplings.

Observing the evolution of the topological order parameter as the magnetic field increases, which we define as the chirality per sublattice and which can be in turn associated with the topological charge or number of skyrmions, we observe a clear jump, indicating a discontinuous transition from a spiral phase into the AF-SkX phase, where multiple Bragg peaks coexist in the spin structure factor. For higher fields, a second (probably continuous) transition occurs into a featureless paramagnetic phase.

We also observe that after a magnetic field quench, the skyrmion number persists for longer MC times as one should have expected, making apparent the robustness of the AF-SkX state. The relation of this AF-SkX state with the 3-sublattice structure of the lattice studied in the present paper is contrasted with the square lattice case, where such a state does not show up. Finally, we have also shown that doped electrons that would evolve in this background would feel a fictitious nonzero magnetic flux, as depicted in the central panel of Fig. 4. Interestingly, the total chirality, which is directly related to the local flux felt by the doped electrons, also remains quite constant in agreement with the fact that the AF-SkX structure is remarkably stable for a wide range of magnetic fields, and this of course can have very interesting consequences in the context of the anomalous Hall effect.

\section{ACKNOWLEDGMENTS}

P.P. acknowledges Tsuyoshi Okubo for enlightening discussions. H.D.R. and D.C.C. are partially supported by PIP 0747 CONICET and PICT 1724. 


\section{APPENDIX: MATRIX ELEMENTS FOR SPHERICAL APPROXIMATION}

The explicit expressions for the $3 \times 3$ matrices of the matrix (4) are given by

$$
m_{12}(\mathbf{k})=\left[J_{12}^{\alpha \beta}\right], m_{13}(\mathbf{k})=\left[J_{13}^{\alpha \beta}\right], m_{32}(\mathbf{k})=\left[J_{32}^{\alpha \beta}\right],
$$

where

$$
\begin{gathered}
J_{12}^{\alpha \beta}=\left(1+\gamma_{1, \mathbf{k}}^{*}+\gamma_{2, \mathbf{k}}^{*}\right) \delta^{\alpha \beta}+\kappa \sum_{\gamma=1}^{2}\left(\vec{e}_{1}-\gamma_{1, \mathbf{k}}^{*} \vec{e}_{2}-\gamma_{2, \mathbf{k}}^{*} \vec{e}_{3}\right)^{\gamma} \varepsilon^{\alpha \beta \gamma}, \\
J_{13}^{\alpha \beta}=\left(1+\gamma_{1, \mathbf{k}}^{*} \gamma_{2, \mathbf{k}}+\gamma_{1, \mathbf{k}}^{*}\right) \delta^{\alpha \beta}+\kappa \sum_{\gamma=1}^{2}\left(\vec{e}_{2}+\gamma_{1, \mathbf{k}}^{*} \gamma_{2, \mathbf{k}} \vec{e}_{3}-\gamma_{1, \mathbf{k}}^{*} \vec{e}_{1}\right)^{\gamma} \varepsilon^{\alpha \beta \gamma}, \\
J_{32}^{\alpha \beta}=\left(1+\gamma_{1, \mathbf{k}} \gamma_{2, \mathbf{k}}^{*}+\gamma_{2, \mathbf{k}}^{*}\right) \delta^{\alpha \beta}+\kappa \sum_{\gamma=1}^{2}\left(\vec{e}_{3}+\gamma_{1, \mathbf{k}} \gamma_{2, \mathbf{k}}^{*} \vec{e}_{2}-\gamma_{2, \mathbf{k}}^{*} \vec{e}_{1}\right)^{\gamma} \varepsilon^{\alpha \beta \gamma},
\end{gathered}
$$

where $\vec{e}_{1}=(1,0), \vec{e}_{2}=(1 / 2, \sqrt{(3)} / 2), \vec{e}_{3}=\vec{e}_{1}-\vec{e}_{2}$ and $\gamma_{1, \mathbf{k}}=e^{-i \mathbf{k} \cdot\left(\vec{e}_{1}+\vec{e}_{2}\right)}$ and $\gamma_{2, \mathbf{k}}=e^{-i \mathbf{k} \cdot\left(2 \vec{e}_{1}-\vec{e}_{2}\right)}$.

[1] N. Romming, C. Hanneken, M. Menzel, J. E. Bickel, B. Wolter, K. von Bergmann, A. Kubetzka, and R. Wiesendanger, Science 341, 636 (2013).

[2] S. Mülbauer, B. Binz, F. Jonietz, C. Pfleiderer, A. Rosch, A. Neubauer, R. Georgii, and P. Böni, Science 323, 915 (2009); W. Münzer, A. Neubauer, T. Adams, S. Mühlbauer, C. Franz, F. Jonietz, R. Georgii, P. Böni, B. Pedersen, M. Schmidt, A. Rosch, and C. Pfleiderer, Phys. Rev. B 81, 041203(R) (2010); X. Z. Yu, Y. Onose, N. Kanazawa, J. H. Park, J. H. Han, Y. Matsui, N. Nagaosa, and Y. Tokura, Nature (London) 465, 901 (2010).

[3] A. N. Bogdanov and D. A. Yablonskii, Sov. Phys. JETP 68, 101 (1989); A. Bogdanov and A. Hubert, J. Magn. Magn. Mater. 138, 255 (1994).

[4] U. K. Rößler, A. N. Bogdanov, and C. Pfleiderer, Nature (London) 442, 797 (2006).

[5] S. D. Yi, S. Onoda, N. Nagaosa, and J. H. Han, Phys. Rev. B 80, 054416 (2009).

[6] D. Solenov, D. Mozyrsky, and I. Martin, Phys. Rev. Lett. 108, 096403 (2012).

[7] X. Z. Yu, N. Kanazawa, Y. Onose, K. Kimoto, W. Z. Zhang, S. Ishiwata, Y. Matsui, and Y. Tokura, Nat. Mater. 10, 106 (2011).
[8] S. Heinze, K. von Bergmann, M. Menzel, J. Brede, A. Kubetzka, R. Wiesendanger, G. Bihlmayer, and S. Blügel, Nat. Phys. 7, 713 (2011).

[9] T. Okubo, S. Chung, and H. Kawamura, Phys. Rev. Lett. 108, 017206 (2012).

[10] M. E. Zhitomirsky, Phys. Rev. Lett. 88, 057204 (2002); L. E. Svistov, A. I. Smirnov, L. A. Prozorova, O. A. Petrenko, L. N. Demianets, and A. Ya. Shapiro, Phys. Rev. B 67, 094434 (2003).

[11] M. V. Gvozdikova, P.-E. Melchy, and M. E. Zhitomirsky, J. Phys.: Condens. Matter 23, 164209 (2011); L. Seabra, T. Momoi, P. Sindzingre, and N. Shannon, Phys. Rev. B 84, 214418 (2011).

[12] J. Villain, R. Bidaux, J.-P. Carton, and R. Conte, J. Phys. 41, 1263 (1980); E. Shender, Sov. Phys.-JETP 56, 178 (1982).

[13] T. Nagamiya, in Solid State Physics, edited by F. Seitz, D. Tumbull, and H. Ehrenreich (Academic Press, New York, 1967), Vol. 20, p. 305.

[14] P. W. Anderson and H. Hasegawa, Phys. Rev. 100, 675 (1955).

[15] K. Ohgushi, S. Murakami, and N. Nagaosa, Phys. Rev. B 62, R6065 (2000).

[16] J. H. Han, J. Zang, Z. Yang, J.-H. Park, and N. Nagaosa, Phys. Rev. B 82, 094429 (2010). 